\title{
Research Paper: Investigating the Psychometric Properties of the 8-Item Somatic Symptom Scale in Non-clinical Sample of Iranian People
}

\author{
Mana Goodarzi $^{1}$, , Seyed Mojtaba Ahmadi², Maryam Asle Zaker Lighvan ${ }^{1}$, Farima Rahmati ${ }^{1}$, Mohammadreza Molavi ${ }^{3}$, Mohammad \\ Mohammadi $^{1 *} \mathrm{C}$
}

1. Department of Clinical Psychology, School of Medicine, Shahid Beheshti University of Medical Sciences, Tehran, Iran. 2. Department of Clinical Psychology, School of Medicine, Kermanshah University of Medical Sciences, Kermanshah, Iran.

3. Department of Clinical Psychology, University of Social Welfare and Rehabilitation Sciences, Tehran, Iran.

\begin{tabular}{|c|c|}
\hline $\begin{array}{c}\text { Use yur device to scan } \\
\text { and read the article online }\end{array}$ \\
tigating the Psychometric Properties of the 8-Item Somatic Symptom Scale in Non-clinical Sample of Iranian People. Journal of \\
Practice in Clinical Psychology, 8(1), 57-64. https://doi.org/10.32598/jpcp.8.1.59 \\
d. https://doi.org/10.32598/jpcp.8.1.59
\end{tabular}

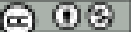

Article info:

Received: 20 Jun 2019

Accepted: 03 Nov2019

Available Online: 01 Jan 2020

Keywords:

Somatic Symptom Disorder (SSD), Somatic symptom burden, Psychometric properties, 8-Item Somatic Symptom Scale (SSS-8)

\section{ABSTRACT}

Objective: Somatic Symptom Disorder (SSD) refers to one or more symptoms that cause distress or a significant disruption in one's daily life and is correlated with poor Quality of Life and psychological distress, as well as an increased burden on health services. Therefore, its identification and treatment are of great importance because of the high prevalence and cost of this syndrome. The present study aimed to evaluate the factor structure, as well as the validity and reliability of the 8-Item Somatic Symptom Scale (SSS-8) in a non-clinical sample of Iranian society.

Methods: The study participants included 281 individuals, who referred to Taleghani Hospital as the patients' companions. After obtaining their informed consent, the participants answered Beck anxiety inventory, general health, SSS- 8 , and somatic symptoms 12 questionnaires. After completing the questionnaires, the collected data were analyzed by SPSS v. 24 and LISREL software.

Results: Confirmatory factor analysis indicated that gastrointestinal problems, pain, fatigue, and cardiopulmonary complaints were the general factor loadings of somatic symptoms. Besides, the 8-item Somatic Symptom Scale had good internal consistency. Regarding validity, the 8-question scale showed a positive correlation with the Beck anxiety inventory $(\mathrm{r}=0.70)$, general health questionnaire $(\mathrm{r}=0.70)$ and, somatic symptoms $12(\mathrm{r}=0.52)$.

Conclusion: The findings provided preliminary support for the validity and reliability of the scores derived from the 8-item Somatic Symptom Scale. This scale can be used to evaluate the severity of somatic symptoms of patients referring to health clinics. Additionally, it is beneficial for interested researchers to investigate this disorder and its complications.

\section{* Corresponding Author:}

Mohammad Mohammadi, PhD.

Address: Department of clinical psychology, School of medicine, Shahid Beheshti University of Medical Sciences, Tehran, Iran.

Tel: +98 (912) 2626035

E-mail: mohammadmohammadi059@gmail.com 


\section{Highlights}

- Somatic symptom disorder causes distress or a significant disruption in one's daily life.

- Its identification and treatment are of great importance because of the high prevalence and cost.

\section{Plain Language Summary}

Somatic symptom disorder refers to symptoms that cause distress or a significant disruption in daily life. It is rrelated with poor Quality of Life and psychological distress, as well as an increased burden on health services. Therefore, its identification and treatment is important because of the high prevalence and cost . The present study aimed to evaluate the factor structure, as well as the validity and reliability of the 8-item somatic symptom scale (SSS-8). Confirmatory factor analysis indicated that gastrointestinal problems, pain, fatigue, and cardiopulmonary complaints were the general factor loadings of somatic symptoms. This scale can be used to evaluate the severity of somatic symptoms of patients referring to health clinics. It is beneficial for interested researchers to investigate this disorder and its complications.

\section{Introduction}

omatic symptoms disorder refers to one or more somatic symptoms that are distressing and result in significant disruption of daily life. Although somatic symptoms are not always present, the state of being symptomatic is persistent (typically more than six months). Most of the time, no medical explanation is found for this disorder (Kop, Toussaint, Mols, \& Löwe, 2019). Common symptoms of this disorder include various types of pain (low back pain, joint pain, headache), gastrointestinal problems (problems with digestion, vomiting, bloating), cardiovascular disorders (sweating, trembling), and chronic fatigue (Henningsen, 2018). The mean prevalence of somatic symptoms was $0.4 \%$ in the general population (Poloni et al., 2019).

Based on another report, $80 \%$ of individuals experience one or more somatic symptoms in any given month (Toussaint, Löwe, Brähler, \& Jordan, 2017). Research has shown that $15 \%-54 \%$ of referrals to specialists are due to somatic symptoms that are categorized as unexplained symptoms (Lehmann et al., 2019). Overall, those who feel distressed from somatic symptoms are investigated in clinical examinations, and $25 \%$ of patients experience persistent somatic symptoms with unknown reasons (Kroenke, 2014, 2016). Physical symptoms are highly comorbid with anxiety and mood disorders, and the burden of health care from physical symptoms is comparable to mood and anxiety disorders (Fujii et al., 2018). Physical symptoms are significantly correlated with lower Quality of Life and psychological distress, as well as increased use of health services (Kohlmann, Gierk, Hilbert, Brähler, \& Löwe, 2016).
Due to the high prevalence and economic and social burden of this syndrome, its identification and treatment are of great importance and necessity. A review study showed that by October 2012, approximately 40 questionnaires were available to measure somatic symptoms. Among them are the Patient Health Questionnaire (PHQ-15), symptom checklist 90, and somatization scale questionnaires with better psychometric properties than the rest had questionnaires (Zijlema et al., 2013). In 2014, Gierke et al. introduced the 8-Item Somatic Symptom Scale (SSS-8) based on the items of the PHQ-15 scale (Gierk et al., 2014).

SSS- 8 is a questionnaire that measures somatic symptoms. It has some advantages over other instruments. For a start, it evaluates different dimensions of the disorder with fewer items. Many comparative studies suggest the use of the SSS-8 as a more efficient alternative to the original PHQ-15, especially in clinical settings or in research settings with limited assessment time to reduce the measurement burden for study participants (Toussaint, Kroenke, Baye, \& Lourens, 2017). For this reason, the need for a precise and appropriate instrument for measuring the burden of physical cues is of paramount importance. The present study aimed to investigate the psychometric properties of the SSS-8 in a non-clinical sample of Iranian society.

\section{Methods}

\section{Study participants}

The study population comprised patients' companions who refer to Tehran hospitals. In this regard, Taleghani Hospital was selected randomly. The study participants included 281 individuals who referred to Taleghani Hospital as the patients' companions. The exclusion criteria 
were suffering from cognitive disorders (such as dementia, Alzheimer disease) and psychological problems (such as psychosis disorder). They were informed about the study objectives, and after completing the questionnaires, the collected data were analyzed by SPSS and LISREL software.

\section{Translation}

For translation and cultural adaptation of the present questionnaire, minimum translation characteristics were used (Trust, 1997). At first, the questionnaire was translated into Persian by two Persian speakers (Master in Clinical Psychology) expert in English. The two translated versions were compared by one evaluator (PhD. in Clinical Psychology). After that, another PhD student in Clinical Psychology translated it back to English. The back-translated questionnaire was quite similar to the original one.

\section{Study instruments}

\section{Beck Anxiety Inventory (BAI)}

Beck developed the Beck Anxiety Inventory (BAI) in 1988. This inventory contains 21 items that investigate the signs and symptoms of anxiety. The participant can choose one of the four options ("never=0", "mild=1", "moderate $=2$ " and "severe $=3$ ") to describe the severity of his or her anxiety. Therefore, the total score of this inventory ranges from 0 to 63 . Each of the items in the questionnaire describes one of the most common symptoms of anxiety (mental, physical, and phobic symptoms). The Cronbach $\alpha$ and test-retest reliability of this inventory were examined and found 0.92 and 0.76 , respectively (Beck, Epstein, Brown, \& Steer, 1988). In a validation study in Iran, the Cronbach $\alpha$ was reported 0.90 . Five subscales were obtained using factor analysis, which explained $58.54 \%$ of the scores of the inventory (Rafiei, \& Akram , 2013). The internal consistency of this questionnaire was 0.86 (Cronbach $\alpha=0.86$ ).

\section{General Health Questionnaire (GHQ-28)}

The General Health Questionnaire (GHQ-28) was compiled by Goldberg to measure apathy, medical discomfort, and general health over the past month. It aimed to detect minor levels of psychiatric disturbance in the general population and consist of items relating to depression, self-confidence, and problem-solving. The questionnaire contains 28 questions with answers rated based on a Likert-type scale. The lowest and highest scores are 0 and 56 . It has good internal consistency
(Cronbach $\alpha=0.79)$ and reliability (0.91). Besides, in Iran, the internal consistency was calculated to be 0.76 using the Cronbach $\alpha$ coefficient. Also, it is highly correlated with similar scales (Abdolmohammadi, Saadat Seyed Pourmand, N, Falsafinejad, 2018). The internal consistency of GHQ-28 in this study based on the Cronbach $\alpha$ was 0.93 .

\section{The scale of Somatic Symptom Disorder (SSD)}

The SSS-8 is an abbreviated 8-item version of the PHQ-15 developed by Gierk et al. according to the DSM-5 field trials. The items of this scale assess the gastrointestinal problems, headaches, joint pain, dizziness, and difficulty falling asleep and are scored based on a 5 -point Likert-type scale. The factor structure reflects gastrointestinal problems, pain, fatigue, and cardiopulmonary aspects of the general somatic symptom burden. The SSS-8 had good reliability (Cronbach $\alpha=0.81$ ) (Gierk et al., 2014).

\section{The Somatic Symptom Disorder (SSD-12)}

The SSD-12 was designed to assess the psychological features of DSM-5 somatic symptom disorder. It has excellent reliability (Cronbach $\alpha=0.95$ ). Confirmatory factor analyses revealed a high correlation between the three suggested psychological subscales defined as cognitive, affective, and behavioral aspects, designating a general factor model of the SSD-12 in the general population $(\mathrm{n}=2362, \mathrm{CFI}=0.99, \mathrm{TLI}=0.998, \mathrm{RMSEA}=0.09$, 90\% CI: 0.09-0.1) (Toussaint, Kroenke, et al., 2017). The internal consistency (Cronbach $\alpha$ ) of SSD-12 in this study was 0.87 .

\section{Results}

The study participants included 281 individuals who referred to Taleghani Hospital as the patients' companions. The mean age of the sample was 24.2 years, and $65.1 \%$ of them were women. About $24.2 \%$ had a high school education or lower, $42.3 \% \mathrm{BS}, 25.3 \% \mathrm{MS}$, and $8.2 \% \mathrm{PhD}$. Also, $86.5 \%$ of them were single.

\section{Reliability and analysis of items}

The internal consistency of the SSS-8 questionnaire based on the Cronbach $\alpha$ was 0.75 , and the correlation between items in the questionnaire was 0.08 to 0.38 . The results also showed that if the questionnaire items were removed, the $\alpha$ level would decrease (Table 1). 
The internal consistency values based on the Cronbach $\alpha$ for pain, cardiopulmonary, and fatigue subscales were $0.63,0.50$, and 0.55 , respectively.

The correlation between the total score of the SSS-8 scale with the BAI, patient health scale, and SSD-12 were $0.70,0.64$, and 0.52 , respectively, all of which were significant (Table 3).

To assess the factor structure of the introduced model, we used LISREL and higher-order factor analysis. It has revealed good fit indices for the higher-order general factor model $\left(\chi^{2} / \mathrm{df}=2.33\right.$, comparative fit index $[\mathrm{CFI}]=$ 0.96 , root mean square error of approximation [RM$\mathrm{SEA}]=0.069$ ). In the first investigation by designer, this scale has four subscales, and the first question is the in- dependent subscale evaluated gastrointestinal problems. Accordingly, the current model is similar to previously introduced models.

\section{Discussion}

The present study aimed to evaluate the factor structure of the SSS-8, as well as its validity and reliability in the non-clinical sample of Iranian society. The data analysis showed that it has acceptable psychometric properties (reliability and validity). The internal consistency, calculated based on the Cronbach $\alpha$ for the whole scale was 0.75 , and for the pain, cardiopulmonary, and fatigue subscales were $0.63,0.50$, and 0.55 , respectively. The present results are in line with the results of a study by

Table 1. Item analysis of SSS-8

\begin{tabular}{|cccccc}
\hline Items & $\begin{array}{c}\text { Scale Mean if } \\
\text { Item Deleted }\end{array}$ & $\begin{array}{c}\text { Scale Variance if } \\
\text { Item Deleted }\end{array}$ & $\begin{array}{c}\text { Corrected Item- } \\
\text { Total Correlation }\end{array}$ & $\begin{array}{c}\text { Squared Multiple } \\
\text { Correlations }\end{array}$ & $\begin{array}{c}\text { Cronbach } \boldsymbol{\alpha} \text { if } \\
\text { Item Deleted }\end{array}$ \\
\hline SSS-1 & 14.01 & 21.06 & 0.32 & 0.15 & 0.75 \\
\hline SSS-2 & 14.17 & 20.06 & 0.51 & 0.31 & 0.72 \\
\hline SSS-3 & 14.12 & 19.68 & 0.48 & 0.33 & 0.72 \\
\hline SSS-4 & 14.04 & 19.77 & 0.49 & 0.26 & 0.72 \\
\hline SSS-5 & 14.60 & 21.01 & 0.50 & 0.28 & 0.73 \\
\hline SSS-6 & 14.55 & 21.42 & 0.45 & 0.24 & 0.73 \\
\hline SSS-7 & 13.19 & 18.28 & 0.52 & 0.29 & 0.72 \\
\hline SSS-8 & 13.64 & 19.14 & 0.42 & 0.22 & 0.74 \\
\hline
\end{tabular}

Table 2. Correlations among each item and other items belonging to the same or different symptom domains

\begin{tabular}{|ccccc|}
\hline \multicolumn{1}{c}{ SSS-8 } & \multicolumn{3}{c}{ Domain } \\
\cline { 2 - 5 } & $\begin{array}{c}\text { Gastrointestinal } \\
\text { Item } \# \mathbf{1}\end{array}$ & $\begin{array}{c}\text { Pain } \\
\text { Item \#2-4 }\end{array}$ & $\begin{array}{c}\text { Cardiopulmonary } \\
\text { Item \#5-6 }\end{array}$ & $\begin{array}{c}\text { Fatigue } \\
\text { Item \#7-8 }\end{array}$ \\
\hline 1. Stomach or bowel problems & 1 & 0.28 & 0.29 & 0.21 \\
2. Back pain & 0.23 & 0.77 & 0.41 & 0.33 \\
\hline 3. Pain in your arms, legs, or joints & 0.15 & 0.79 & 0.37 & 0.35 \\
4. Headache & 0.25 & 0.71 & 0.39 & 0.39 \\
\hline 5. Dizziness & 0.27 & 0.45 & 0.82 & 0.34 \\
\hline 7. Feeling tired or having low energy & 0.21 & 0.38 & 0.81 & 0.35 \\
\hline 8. Trouble sleeping & 0.28 & 0.41 & 0.23 & 0.82 \\
\hline
\end{tabular}


Table 3. Correlation of total score and subscales of SSS-8 test with Beck Anxiety, Patient Health Questionnaire and SSD-12

\begin{tabular}{|c|c|c|c|c|c|c|c|}
\hline Variables & SSS-8 & BAI & $\begin{array}{l}\text { General Health } \\
\text { Questionnaire }\end{array}$ & SSD-12 & $\begin{array}{l}\text { SSS- } \\
\text { 8_pain }\end{array}$ & $\begin{array}{c}\text { SSS-8_- } \\
\text { Cardiopulmonary }\end{array}$ & $\begin{array}{l}\text { SSS-8_ } \\
\text { Fatigue }\end{array}$ \\
\hline SSS-8 & 1 & & & & & & \\
\hline $\mathrm{BAl}$ & $0.704 *$ & 1 & & & & & \\
\hline $\begin{array}{l}\text { General health } \\
\text { questionnaire }\end{array}$ & $0.643^{*}$ & $0.696^{*}$ & 1 & & & & \\
\hline SSD-12 & $0.521^{*}$ & $0.538^{*}$ & $0.481^{*}$ & 1 & & & \\
\hline SSS-8_pain & $0.843^{*}$ & $0.557^{*}$ & 0.502 & $0.460^{*}$ & 1 & & \\
\hline SSS-8_Cardiopulmonary & $0.730^{*}$ & $0.589 *$ & $0.467^{*}$ & $0.430 *$ & $0.516^{*}$ & 1 & \\
\hline SSS-8_Fatigue & $0.783 *$ & $0.567^{*}$ & $0.590 *$ & $0.379 *$ & $0.475^{*}$ & $0.427^{*}$ & 1 \\
\hline
\end{tabular}

Table 4. Fit indices of the model

\begin{tabular}{|c|c|c|c|c|c|c|c|}
\hline $\mathbf{P}$ & RMSEA* & GFI* & $\mathrm{CFI}^{*}$ & $\chi^{2} / d f$ & df & $\chi^{2}$ & Model \\
\hline 0.001 & 0.069 & 0.970 & 0.96 & 2.33 & 17 & 39.75 & Three factors \\
\hline
\end{tabular}

* CFI: Comparative fit index; GFI: Goodness of fit index; RMSEA: Root mean square error of approximation

Benjamin Gierk et al. that obtained the Cronbach $\alpha$ of 0.81 for the whole items (Gierk et al., 2014).

Also, the concurrent validity analysis of the SSS-8 scale showed a significant correlation (between 0.52 and 0.7) with other mental health scales such as GHQ-28, BAI, and SSD-12. In line with the previous study that
The SSS-8 was designed to be an abbreviated version of the established PHQ-15, a study in 2008 by Bernd Löwe showed a significant relationship between somatic burden and mental health components such as anxiety. In other words, SSS-8 has convergent validity with mental health scales (Löwe et al., 2008).

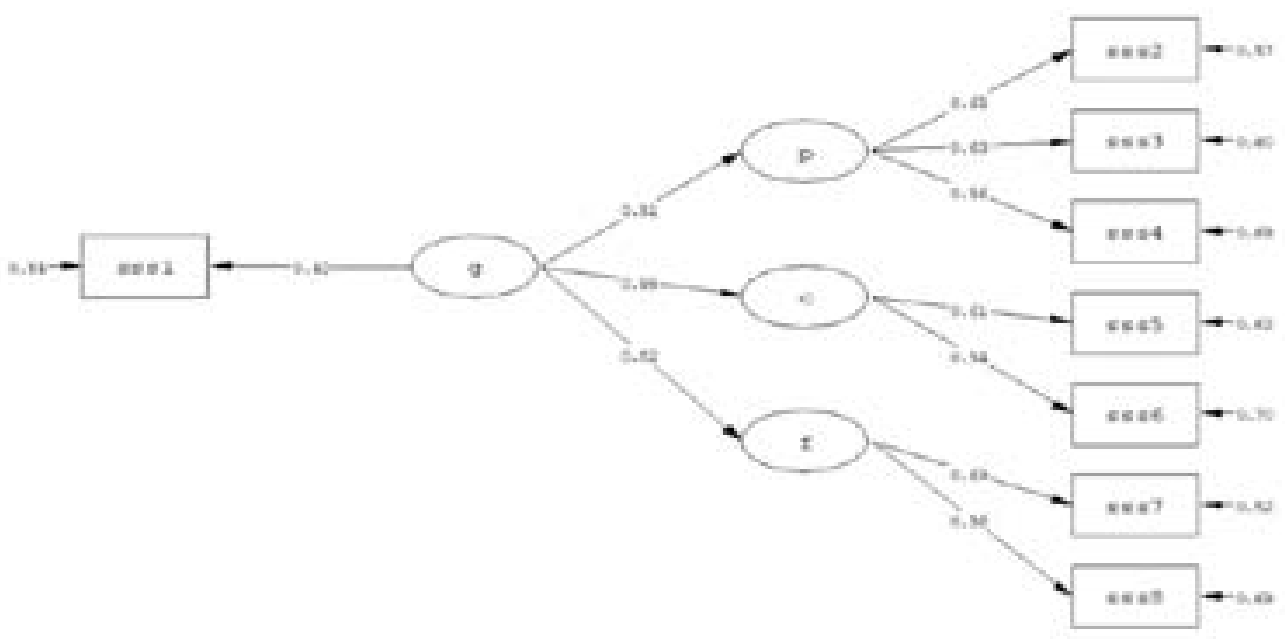

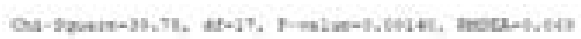

Figure 1. Confirmatory factor analysis of the higher-order general-factor model

P: Pain; C: Cardiopulmonary; F: Fatigue; SSS-1: Somatic Symptom Scale; Gastrointestinal; g: General factor 
Based on the factor analysis results, the higher-order general-factor structure revealed its acceptable fit for the four-factor model. Similarly, the subscales have identified in the previous report by Gierke et al. The model under study in which defined four components as a general factor: pain, gastrointestinal symptoms, cardiopulmonary symptoms, and fatigue. Also, a high correlation was found between each subscale and other subscales items so that the items on pain subscale with the total score of this subscale were between 0.71 to 0.79 and in two cardiopulmonary and fatigue subscales were 0.81 to 0.82 and 0.82 to 0.83 , respectively. The results are in line with the results of Matsudaira et al. (2017). In this study conducted in Japan, the results showed that the SSS-8 scale has convergent validity as well as discriminant validity, which is similar to the results obtained in the present study (Matsudaira et al., 2017). From a clinical viewpoint, these clusters correspond to 4 common medical syndromes.

This scale needs further research in several groups of participants with different measurements because different ages and sexes influence it. Also, it is expected that future studies will demonstrate that SSS-8 is useful in assessing changes in the severity of somatic symptoms over time.

\section{Conclusion}

In general, SSS-8 has acceptable psychometric properties. It most probably is beneficial in many medical settings. The scores could serve to evaluate somatic symptom burden in patients with chronic diseases, such as cancer or coronary heart disease. Additionally, it is beneficial for interested researchers in investigating this disorder and associated outcomes.

\section{Ethical Considerations}

\section{Compliance with ethical guidelines}

All ethical principles were considered in this article. The participants were informed about the purpose of the research and its implementation stages; they were also assured about the confidentiality of their information; Moreover, They were allowed to leave the study whenever they wish, and if desired, the results of the research would be available to them.

\section{Funding}

This research did not receive any specific grant from funding agencies in the public, commercial, or not-forprofit sectors.

\section{Authors' contributions}

All authors contributed equally in preparing all parts of the research.

\section{Conflict of interest}

The authors declared no conflict of interest.

\section{References}

Abdolmohammadi, K. G., F. Saadat Seyed Pourmand, N, Falsafinejad, M. (2018). The validation of Somatization Inventory in the Students of Tabriz universities. Qom university medical science, 11(12), 61-7.

Beck, A. T., Epstein, N., Brown, G., \& Steer, R. A. (1988). An inventory for measuring clinical anxiety: Psychometric properties. Journal of consulting and clinical psychology, 56(6), 893. [DOI:10.1037/0022-006X.56.6.893] [PMID]

Fujii, T., Oka, H., Katsuhira, J., Tonosu, J., Kasahara, S., Tanaka, S., \& Matsudaira, K. (2018). Association between somatic symptom burden and health-related Quality of Life in people with chronic low back pain. PloS one, 13(2), e0193208. [DOI:10.1371/journal.pone.0193208] [PMID] [PMCID]

Gierk, B., Kohlmann, S., Kroenke, K., Spangenberg, L., Zenger, M., Brähler, E., \& Löwe, B. (2014). The somatic symptom scale-8 (SSS-8): A brief measure of somatic symptom burden. JAMA internal medicine, 174(3), 399-407. [DOI:10.1001/jamainternmed.2013.12179] [PMID]

Henningsen, P. (2018). Management of somatic symptom disorder. Dialogues in Clinical Neuroscience, 20(1), 23.

Kohlmann, S., Gierk, B., Hilbert, A., Brähler, E., \& Löwe, B. (2016). The overlap of somatic, anxious and depressive syndromes: A population-based analysis. Journal of psychosomatic research, 90, 51-6. [DOI:10.1016/j.jpsychores.2016.09.004] [PMID]

Kop, W. J., Toussaint, A., Mols, F., \& Löwe, B. (2019). Somatic symptom disorder in the general population: Associations with medical status and health care utilization using the SSD12. General hospital psychiatry, 56, 36-41. [DOI:10.1016/j.genhosppsych.2018.10.004] [PMID]

Kroenke, K. (2014). A practical and evidence-based approach to common symptoms: a narrative review. Annals of internal medicine, 161(8), 579-86. [DOI:10.7326/M14-0461] [PMID]

Lehmann, M., Jonas, C., Pohontsch, N. J., Zimmermann, T. Scherer, M., \& Löwe, B. (2019). General practitioners' views on the diagnostic innovations in DSM-5 somatic symptom disorder-A focus group study. Journal of psychosomatic research, 123, 109734. [DOI:10.1016/j.jpsychores.2019.109734] [PMID]

Löwe, B., Spitzer, R. L., Williams, J. B., Mussell, M., Schellberg, D. \& Kroenke, K. (2008). Depression, anxiety and somatization in primary care: Syndrome overlap and functional impairment. General hospital psychiatry, 30(3), 191-9. [DOI:10.1016/j. genhosppsych.2008.01.001] [PMID] 
Matsudaira, K., Oka, H., Kawaguchi, M., Murakami, M., Fukudo, S., Hashizume, M., \& Löwe, B. (2017). Development of a Japanese version of the Somatic Symptom Scale-8: Psychometric validity and internal consistency. General hospital psychiatry, 45, 7-11. [DOI:10.1016/j.genhosppsych.2016.12.002] [PMID]

Poloni, N., Caselli, I., Ielmini, M., Mattia, M., De Leo, A., Di Sarno, M., et al. (2019). Hospitalized Patients with Medically Unexplained Physical Symptoms: Clinical Context and Economic Costs of Healthcare Management. Behavioral Sciences, 9(7), 80. [DOI:10.3390/bs9070080] [PMID] [PMCID]

Rafiei, M. S., Akram. (2013). An Investigation into reliabiliy and Validity of Beck Anxiety Inventory among the university Students. Thought and Behavior in Clinical Psychology, 7(27).

Toussaint, A., Kroenke, K., Baye, F., \& Lourens, S. (2017). Comparing the Patient Health Questionnaire-15 and the Somatic Symptom Scale-8 as measures of somatic symptom burden. Journal of psychosomatic research, 101, 44-50. [DOI:10.1016/j. jpsychores.2017.08.002] [PMID]

Toussaint, A., Löwe, B., Brähler, E., \& Jordan, P. (2017). The Somatic Symptom Disorder-B Criteria Scale (SSD-12): Factorial structure, validity and population-based norms. Journal of psychosomatic research, 97, 9-17. [DOI:10.1016/j.jpsychores.2017.03.017] [PMID]

Trust, M. (1997). Trust introduces new translation criteria. Medical Outcomes Trust Bulletin, 5, 3-4.

Zijlema, W. L., Stolk, R. P., Löwe, B., Rief, W., White, P. D., \& Rosmalen, J. G. (2013). How to assess common somatic symptoms in large-scale studies: A systematic review of questionnaires. Journal of psychosomatic research, 74(6), 459-68.

[DOI:10.1016/j.jpsychores.2013.03.093] [PMID] 
This Page Intentionally Left Blank 